\title{
Vibration of gold nanobeam with variable thermal conductivity: state-space approach
}

\author{
H. M. Youssef
}

Received: 25 April 2012/ Accepted: 17 September 2012/Published online: 7 October 2012

(C) The Author(s) 2012. This article is published with open access at Springerlink.com

\begin{abstract}
The non-Fourier effect in heat conduction and the coupling effect between temperature and strain rate are the two significant effects in the nanoscale beam. In the present work, the solution of vibration of gold nanobeam resonator induced by thermal shock is developed in the context of generalized thermoelasticity with variable thermal conductivity. State-space and Laplace transform methods are used to determine the lateral vibration, the temperature, the displacement, the strain, the stress, and the strain-stress energy. The numerical results have been studied and represented graphically with some comparisons to stand on the effects of the variability of thermal conductivity.
\end{abstract}

Keywords Thermoelasticity - Euler-Bernoulli equation · Gold nanobeam $\cdot$ State-space approach

\section{Introduction}

Many attempts have been made to investigate the elastic properties of nanostructured materials using atomistic simulations. Diao et al. (2004) studied the effect of free surfaces on the structure and elastic properties of gold nanowires by atomistic simulations. Although the atomistic simulation is a good way to calculate the elastic constants of nanostructured materials, it is only applicable to

H. M. Youssef $(\bowtie)$

Mechcanical Department, Faculty of Engineering,

Umm Al-Qura University, P.O. Box 5555, Makkah,

Saudi Arabia

e-mail: yousefanne@yahoo.com

H. M. Youssef

Mathematical Department, Faculty of Education,

Alexandria University, Alexandria, Egypt homogeneous nanostructured materials (e.g., nanoplates, nanobeams, nanowires, etc.) with limited number of atoms. Moreover, it is difficult to obtain the elastic properties of the heterogeneous nanostructured materials using atomistic simulations. For these and other reasons, it is prudent to seek a more practical approach. One such approach would be to extend the classical theory of elasticity down to the nanoscale by including in it the hitherto neglected surface/ interface effect. For this it is necessary first to cast the latter within the framework of continuum elasticity.

Recently and due to their many important technological applications, nanomechanical resonators have attracted considerable attention. Accurate analysis of various effects on the characteristics of resonators, such as resonant frequencies and quality factors, is crucial for designing highperformance components. Many authors have studied the vibration and heat transfer process of beams. Kidawa (2003) has studied the problem of transverse vibrations of a beam induced by a mobile heat source. The analytical solution to the problem was obtained using the Green's functions method. However, Kidawa did not consider the thermoelastic coupling effect. Boley (1972) analyzed the vibrations of a simply supported rectangular beam subjected to a suddenly applied heat input distributed along its span. Manolis and Beskos (1980) examined the thermally induced vibration of structures consisting of beams, exposed to rapid surface heating. They have also studied the effects of damping and axial loads on the structural response. Al-Huniti et al. (2001) investigated the thermally induced displacements and stresses of a rod using the Laplace transformation technique. Ai Kah Soh et al. (2008) studied the vibration of micro/nanoscale beam resonators induced by ultra-short-pulsed laser by considering the thermoelastic coupling term in Sun and Fang (2008). The propagation characteristics of the longitudinal wave in 
nanoplates with small-scale effects are studied by Wang et al. (2010).

The uncoupled theory of thermoelasticity (the classical thermoelasticity) predicts two phenomena not compatible with physical observations. First, the equation of heat conduction of this theory does not contain any elastic terms. Second, the heat equation is of a parabolic type, predicting infinite speeds of propagation for heat waves. Biot (1956) introduced the theory of coupled thermoelasticity to overcome the first shortcoming. The governing equations for this theory are coupled, eliminating the first paradox of the classical theory. However, both theories share the second shortcoming since the governing equations for the coupled are of the mixed: parabolic-hyperbolic. When very fast phenomena and small structure dimensions are involved, the classical law of Fourier becomes inaccurate. Modern technology has enabled the fabrication of materials and devices with characteristic dimensions of a few nanometers. Examples are super lattices, nanowires, and quantum dots. At these length scales, the familiar continuum Fourier law for heat conduction is expected to fail due to both classical and quantum size effects (Rao 1992). The generalizations to the coupled theory were introduced by Lord and Shulman (1967), who obtained a wave-type heat equation by postulating a new law of heat conduction to replace the classical Fourier's law. Since the heat equation of this theory is of the wave-type, it automatically ensures finite speeds of propagation for heat and elastic waves. The remaining governing equations for this theory, namely, the equations of motion and constitutive relations, remain the same as those for the coupled and the uncoupled theories. Among many applications, the studying of the thermoelastic damping in MEMS/ NEMS has been improved in Sun et al. (2006), Fang et al. (2006), and Duwel et al. 2003). A more sophisticated model is then needed to describe the thermal conduction mechanisms in a physically acceptable way.

The physical property of a solid body related to application of heat energy is defined as a thermal property. Thermal properties explain the response of a material to the application of heat, and thermal conductivity is one of the most important thermal properties. Thermal conductivity $K$ is ability of a material to transport heat energy through it from high-temperature region to low-temperature region and it is a microstructure sensitive property whose values range from 20 to 400 for metals, from 2 to 50 for ceramics, and for polymers an order of 0.3 . Heat is transported in two ways: electronic contribution and vibrational (phonon) contribution. In metals, electronic contribution is very high. Thus metals have higher thermal conductivities. It is same as electrical conduction. Both conductivities are related through Wiedemann-Franz law: $K=v L T$ where $L$ is Lorentz constant and $v$ is the electrical conductivity. As different contributions to conduction vary with temperature, the above relation is valid to a limited extension for many metals. With increase in temperature, both number of carrier electrons and contribution of lattice vibrations increase. Thus thermal conductivity of a metal is expected to increase. However, because of greater lattice vibrations, electron mobility decreases. The combined effect of these factors leads to very different behavior for different metals. For example, thermal conductivity of iron initially decreases and then increases slightly; thermal conductivity decreases with increase in temperature for aluminum, whereas it increases for platinum and gold (Zhang 2007). The question as to the effects of these variations on the lateral vibration, the temperature, the displacement, the strain, the stress, and the strain-stress energy distributions in nanobeam resonator arises.

Youssef used the state-space approach to solve a problem of generalized thermoelasticity for an infinite material with a spherical cavity and variable thermal conductivity subjected to ramp-type heating (Zhang 2007). The effect of variability of the thermal conductivity on all the studied fields is discussed in Youssef and El-Bary (2006a, b, c) and Ezzat and Youssef (2009). Youssef and Elsibai discussed the vibration of gold nanobeam induced by different types of thermal loading (Youssef and Elsibai 2010).

In particular, the state-space approach is useful because (1) linear systems with time-varying parameters can be analyzed in essentially the same manner as time-invariant linear systems, (2) problems formulated by state-space methods can easily be programmed on a computer, (3) highorder linear systems can be analyzed, (4) multiple inputmultiple output systems can be treated almost as easily as single input-single output linear systems, and (5) state-space theory is the foundation for further studies in such areas as nonlinear systems, stochastic systems, and optimal control (Ezzat 2008). For solving coupled thermoelastic problems using the state-space approach in which the problem is rewritten in terms of state-space variables, namely, the temperature, the displacement and their gradients, has been developed by Bahar and Hetnarski (1977a, b, 1978).

In the present work, the solution of vibration of gold nanobeam resonator induced by thermal shock is developed in the context of generalized thermoelasticity with variable thermal conductivity. State-space and Laplace transform methods are used to determine the lateral vibration, the temperature, the displacement, the strain, the stress, and the strain-stress energy. The effect of the variability of thermal conductivity has been studied and represented graphically with some comparisons.

\section{Formulation of the problem}

Although challenging, creating a beam with a rectangular cross section is the easiest when compared with other cross 


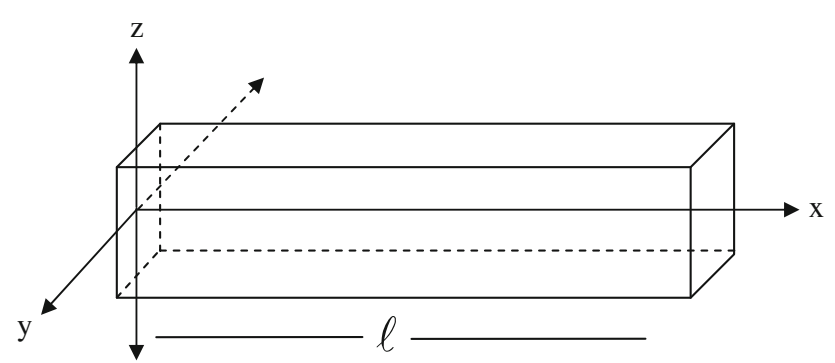

Fig. 1 The gold nanobeam resonator

sections. Consider small flexural deflections of a thin elastic beam of length $\ell(0 \leq x \leq \ell)$, width $b\left(-\frac{b}{2} \leq y \leq \frac{b}{2}\right)$ and thickness $h\left(-\frac{h}{2} \leq z \leq \frac{h}{2}\right)$ as in Fig. 1 for which the $x$, $y$, and $z$ axes are defined along the longitudinal, width, and thickness directions of the beam, respectively. In equilibrium, the beam is unstrained, unstressed, no damping mechanism present, and at temperature $T_{0}$ everywhere (Youssef and Elsibai 2010).

In the present study, the usual Euler-Bernoulli assumption (Youssef and Elsibai 2010) is adopted, i.e., any plane cross-section, initially perpendicular to the axis of the beam remains plane and perpendicular to the neutral surface during bending. Thus, the displacement components $(u, v, w)$ are given by

$$
\begin{aligned}
& u(x, y, z, t)=-z \frac{\partial w(x, t)}{\partial x}, v(x, y, z, t)=0 \\
& \text { and } w(x, y, z, t)=w(x, t) .
\end{aligned}
$$

Hence, the differential equation of thermally induced lateral vibration of the beam may be expressed in the following form (Youssef and Elsibai 2010):

$\frac{\partial^{4} w}{\partial x^{4}}+\frac{\rho A}{E I} \frac{\partial^{2} w}{\partial t^{2}}+\alpha_{T} \frac{\partial^{2} M_{\mathrm{T}}}{\partial x^{2}}=0$,

where $E$ is Young's modulus, $I$ [ $\left.=b h^{3} / 12\right]$ the inertial moment about $x$ axis, $\rho$ the density of the beam, $\alpha_{\mathrm{T}}$ the coefficient of linear thermal expansion, $w(x, t)$ the lateral deflection, $x$ the distance along the length of the beam, $A=h b$ is the cross section area and $t$ the time, and $M_{\mathrm{T}}$ is the thermal moment, which is defined as

$M_{\mathrm{T}}(x, z, t)=\frac{12}{h^{3}} \int_{-h / 2}^{h / 2} \theta(x, z, t) z \mathrm{~d} z$,

where $\theta(x, z, t)=\left(T-T_{0}\right)$ is the dynamical temperature increment of the resonator, in which $T(x, z, t)$ is the temperature distribution and $T_{0}$ the environmental temperature.

According to Lord-Shulman model (L-S), the nonFourier heat conduction equation has the following form (Youssef and Elsibai 2010):

$$
\begin{aligned}
& \frac{\partial}{\partial x}\left(K(\theta) \frac{\partial \theta}{\partial x}\right)+\frac{\partial}{\partial z}\left(K(\theta) \frac{\partial \theta}{\partial z}\right) \\
& \quad=\left(1+\tau_{o} \frac{\partial}{\partial t}\right)\left(\rho C_{E}(\theta) \dot{\theta}+\beta T_{0} \dot{e}\right)
\end{aligned}
$$

where $e=\frac{\partial u}{\partial x}+\frac{\partial v}{\partial y}+\frac{\partial w}{\partial z}$ is the volumetric strain, $C_{\mathrm{E}}(\theta)$ is the specific heat with variable temperature at constant volume, $\tau_{\mathrm{o}}$ the thermal relaxation time, $K(\theta)$ the thermal conductivity with variable temperature, $\rho$ the density, $\beta=$ $\frac{E \alpha_{T}}{1-2 v}$ in which $v$ is Poisson's ratio, and the dot above it means partial derivative with respect to time.

Since we have the relation (Zhang 2007)

$\rho C_{E}(\theta)=\frac{K(\theta)}{\kappa}$,

where $\kappa$ is the thermal diffusivity,

Eq. (4) will take the following form:

$$
\begin{aligned}
& \frac{\partial}{\partial x}\left(K(\theta) \frac{\partial \theta}{\partial x}\right)+\frac{\partial}{\partial z}\left(K(\theta) \frac{\partial \theta}{\partial z}\right) \\
& =\left(1+\tau_{0} \frac{\partial}{\partial t}\right)\left(\frac{K(\theta)}{\kappa} \dot{\theta}+\beta T_{0} \dot{e}\right) .
\end{aligned}
$$

Considering the following mapping which converts the nonlinear terms of the temperature to be linear (Zhang 2007; Youssef and El-Bary 2006a, b, c; Ezzat and Youssef 2009)

$\vartheta=\frac{1}{K_{\mathrm{o}}} \int_{0}^{\theta} K(\xi) \mathrm{d} \xi$,

where $K_{\mathrm{o}}$ is the thermal conductivity at the normal case.

Differentiating Eq. (7) with respect to the coordinates $x$ and $z$, respectively, we get

$K(\theta) \frac{\partial \theta}{\partial x}=K_{\mathrm{o}} \frac{\partial \vartheta}{\partial x}$ and $K(\theta) \frac{\partial \theta}{\partial z}=K_{\mathrm{o}} \frac{\partial \vartheta}{\partial z}$.

Differentiating the above equations with respect to the coordinates $x$ and $z$, respectively, we obtain

$K_{\mathrm{o}} \frac{\partial^{2} \vartheta}{\partial x^{2}}=\frac{\partial}{\partial x}\left[K(\theta) \frac{\partial \theta}{\partial x}\right]$ and $K_{\mathrm{o}} \frac{\partial^{2} \vartheta}{\partial z^{2}}=\frac{\partial}{\partial z}\left[K(\theta) \frac{\partial \theta}{\partial z}\right]$.

Differentiating Eq. (7) with respect to time, we get

$K_{\mathrm{o}} \dot{\vartheta}=K(\theta) \dot{\theta}$,

Appling the Eq. (8)-(10) in Eq. (6), we get

$\frac{\partial^{2} \vartheta}{\partial x^{2}}+\frac{\partial^{2} \vartheta}{\partial z^{2}}=\left(\frac{\partial}{\partial t}+\tau_{\mathrm{o}} \frac{\partial^{2}}{\partial t^{2}}\right)\left(\frac{1}{\kappa} \vartheta+\frac{\beta T_{0}}{K_{\mathrm{o}}} e\right)$,

where there is no heat flow across the upper and lower surfaces of the beam, so that $\frac{\partial \theta}{\partial z}=\frac{\partial \vartheta}{\partial z}=0$ at $z= \pm h / 2$. For a very thin beam and assuming the temperature varies in 
terms of a $\sin (p z)$ function along the thickness direction, where $p=\pi / h$, gives (Youssef and Elsibai 2010):

$\vartheta(x, z, t)=\vartheta_{1}(x, t) \sin (p z)$.

Hence, Eq. (2) gives

$$
\frac{\partial^{4} w}{\partial x^{4}}+\frac{\rho A}{E I} \frac{\partial^{2} w}{\partial t^{2}}+\frac{12 \alpha_{T}}{h^{3}} \frac{\partial^{2} \vartheta_{1}}{\partial x^{2}} \int_{-h / 2}^{h / 2} z \sin (p z) \mathrm{d} z=0 .
$$

To get Eq. (14), we used the linearity condition of thermoelasticity such that $\frac{|\theta|}{T_{0}} \ll 1$, which gives

$\frac{\partial^{2} \theta_{1}}{\partial x^{2}} \approx \frac{\partial^{2} \vartheta_{1}}{\partial x^{2}}$

Equation (11) takes the following form:

$$
\begin{aligned}
& \frac{\partial^{2} \vartheta_{1}}{\partial x^{2}} \sin (p z)-p^{2} \vartheta_{1} \sin (p z) \\
& \quad=\left(\frac{\partial}{\partial t}+\tau_{\mathrm{o}} \frac{\partial^{2}}{\partial t^{2}}\right)\left(\frac{1}{\kappa} \vartheta_{1} \sin (p z)-\frac{\beta T_{0}}{K_{\mathrm{o}}} z \frac{\partial^{2} w}{\partial x^{2}}\right) .
\end{aligned}
$$

After doing the integrations, Eq. (13) gives

$\frac{\partial^{4} w}{\partial x^{4}}+\frac{\rho A}{E I} \frac{\partial^{2} w}{\partial t^{2}}+\frac{24 \alpha_{T}}{h \pi^{2}} \frac{\partial^{2} \vartheta_{1}}{\partial x^{2}}=0$.

In Eq (15), we multiply the both sides by $z$ and integrate with respect to $z$ from $-\frac{h}{2}$ to $\frac{h}{2}$; then we obtain

$$
\left(\frac{\partial^{2} \vartheta_{1}}{\partial x^{2}}-p^{2} \vartheta_{1}\right)=\left(\frac{\partial}{\partial t}+\tau_{\mathrm{o}} \frac{\partial^{2}}{\partial t^{2}}\right)\left(\varepsilon \vartheta_{1}-\frac{\beta T_{0} \pi^{2} h}{24 K_{\mathrm{o}}} \frac{\partial^{2} w}{\partial x^{2}}\right)
$$

where $\varepsilon=\frac{1}{\kappa}$.

Now, for simplicity we will use the following nondimensional variables (Youssef and Elsibai 2010):

$\left(x^{\prime}, w^{\prime}, h^{\prime}\right)=\varepsilon c_{\mathrm{o}}(x, w, h),\left(t^{\prime}, \tau_{\mathrm{o}}^{\prime}\right)=\varepsilon c_{\mathrm{o}}^{2}\left(t, \tau_{\mathrm{o}}\right)$,

$\sigma^{\prime}=\frac{\sigma}{E}, \quad \theta_{1}^{\prime}=\frac{\theta_{1}}{T_{\mathrm{o}}}, c_{\mathrm{o}}^{2}=\frac{E}{\rho}$.

Hence, we have

$\frac{\partial^{4} w}{\partial x^{4}}+A_{1} \frac{\partial^{2} w}{\partial t^{2}}+A_{2} \frac{\partial^{2} \vartheta_{1}}{\partial x^{2}}=0$,

and

$\frac{\partial^{2} \vartheta_{1}}{\partial x^{2}}-A_{3} \vartheta_{1}=\left(\frac{\partial}{\partial t}+\tau_{\mathrm{o}} \frac{\partial^{2}}{\partial t^{2}}\right)\left(\vartheta_{1}-A_{4} \frac{\partial^{2} w}{\partial x^{2}}\right)$,

where

$A_{1}=\frac{12}{h^{2}}, A_{2}=\frac{24 \alpha_{t} T_{\mathrm{o}}}{\pi^{2} h}, A_{3}=p^{2}, A_{4}=\frac{\pi^{2} \beta h}{24 K_{\mathrm{o}} \varepsilon}$.

We have dropped the prime for convenience.
Formulation the problem in the Laplace transform domain

Application of the Laplace transform to Eq. (19) and (20) results in the following formula:

$\bar{f}(s)=L[f(t)]=\int_{0}^{\infty} f(t) \mathrm{e}^{-s t} \mathrm{~d} t$.

Hence, for the zero initial conditions of all the states functions, we obtain the following system:

$\frac{\mathrm{d}^{4} \bar{w}}{\mathrm{~d} x^{4}}+A_{1} s^{2} \bar{w}+A_{2} \frac{\mathrm{d}^{2} \bar{\vartheta}_{1}}{\mathrm{~d} x^{2}}=0$,

and

$\frac{\mathrm{d}^{2} \vartheta_{1}}{\mathrm{dx^{2 }}}-A_{3} \bar{\vartheta}_{1}=\left(s+\tau_{\mathrm{o}} s^{2}\right)\left(\bar{\vartheta}_{1}-A_{4} \frac{\mathrm{d}^{2} \bar{w}}{\mathrm{~d} x^{2}}\right)$.

We will consider the function $\bar{\eta}$ as follows:

$\frac{\mathrm{d}^{2} \bar{w}}{\mathrm{~d} x^{2}}=\bar{\eta}$,

then, we have

$\frac{\mathrm{d}^{2} \bar{\vartheta}_{1}}{\mathrm{~d} x^{2}}=\alpha_{1} \bar{\vartheta}_{1}-\alpha_{2} \bar{\eta}$,

and

$\frac{\mathrm{d}^{2} \bar{\eta}}{\mathrm{d} x^{2}}=-\alpha_{3} \bar{w}-\alpha_{4} \bar{\vartheta}_{1}+\alpha_{5} \bar{\eta}$,

where

$$
\begin{aligned}
& \alpha_{1}=\left(A_{3}+s+\tau_{0} s^{2}\right), \alpha_{2}=A_{4}\left(s+\tau_{0} s^{2}\right), \alpha_{3}=A_{1} s^{2}, \\
& \alpha_{4}=A_{2}\left(A_{3}+s+\tau_{0} s^{2}\right), \alpha_{5}=A_{2} A_{4}\left(s+\tau_{0} s^{2}\right) .
\end{aligned}
$$

\section{State-space formulation}

Choosing as a state variable, the functions $\bar{w}, \bar{\vartheta}_{1}, \bar{\eta}, \frac{\mathrm{d} \bar{w}}{\mathrm{~d} x}=$ $\bar{w}^{\prime}, \frac{\mathrm{d} \bar{\vartheta}_{1}}{\mathrm{~d} x}=\bar{\vartheta}_{1}^{\prime}$ and $\frac{\mathrm{d} \bar{\eta}}{\mathrm{d} x}=\bar{\eta}^{\prime}$ in the $x$ direction, Eq. (23)-(25) can be written in matrix form using the Bahar-Hetnarski method (Youssef and Elsibai 2010; Ezzat 2008; Bahar and Hetnarski 1977a, b, 1978):

$\frac{\mathrm{d} \bar{V}(x, s)}{\mathrm{d} x}=A(s) \bar{V}(x, s)$,

where

$\bar{V}(x, s)=\left[\begin{array}{c}\bar{w}(x, s) \\ \bar{\vartheta}_{1}(x, s) \\ \bar{\eta}(x, s) \\ \bar{w}^{\prime}(x, s) \\ \bar{\vartheta}_{1}^{\prime}(x, s) \\ \bar{\eta}^{\prime}(x, s)\end{array}\right]$, 
and

$$
A(s)=\left[\begin{array}{cccccc}
0 & 0 & 0 & 1 & 0 & 0 \\
0 & 0 & 0 & 0 & 1 & 0 \\
0 & 0 & 0 & 0 & 0 & 1 \\
0 & 0 & 1 & 0 & 0 & 0 \\
0 & \alpha_{1} & -\alpha_{2} & 0 & 0 & 0 \\
-\alpha_{3} & -\alpha_{4} & \alpha_{5} & 0 & 0 & 0
\end{array}\right] .
$$

The formal solution of Eq. (26) is given by

$\bar{V}(x, s)=\exp [A(s) \cdot x] \bar{V}(0, s)$,

where

$$
\bar{V}(0, s)=\left[\begin{array}{c}
\bar{w}(0, s) \\
\bar{\vartheta}_{1}(0, s) \\
\bar{\eta}(0, s) \\
\bar{w}^{\prime}(0, s) \\
\bar{\vartheta}_{1}^{\prime}(0, s) \\
\bar{\eta}^{\prime}(0, s)
\end{array}\right] .
$$

The characteristic equation of the matrix $A(s)$ has the form

$k^{6}-l k^{4}+m k^{2}-n=0$,

where

$l=\alpha_{1}+\alpha_{5}, m=\alpha_{1} \alpha_{5}-\alpha_{2} \alpha_{4}+\alpha_{3}$, and $n=\alpha_{1} \alpha_{3}$.

$k_{1}^{2}, k_{2}^{2}$ and $k_{3}^{2}$ are the roots of the characteristic Eq. (30) and satisfy the following relations:

$k_{1}^{2}+k_{2}^{2}+k_{3}^{2}=l$,

$k_{1}^{2} k_{2}^{2}+k_{2}^{2} k_{3}^{2}+k_{1}^{2} k_{3}^{2}=m$,

$k_{1}^{2} k_{2}^{2} k_{3}^{2}=n$.

The Taylor series expansion for the matrix exponential is given by

$\exp [A(s) x]=\sum_{i=0}^{\infty} \frac{[A(s) x]^{i}}{i !}$

Using the Cayley-Hamilton theorem (Youssef and Elsibai 2010; Ezzat 2008; Bahar and Hetnarski 1977a, b, 1978), this infinite series can be truncated to

$$
\begin{aligned}
\exp [A(s) \cdot x]= & L(x, s)=a_{0} I_{6}+a_{1} A+a_{2} A^{2}+a_{3} A^{3} \\
& +a_{4} A^{4}+a_{5} A^{5},
\end{aligned}
$$

where $I_{6}$ is the unit matrix of order 6 and $a_{0}-a_{5}$ are some parameters depending on $s$ and $x$ to be determined.

Using the Cayley-Hamilton theorem again, we obtain

$\exp \left( \pm k_{i} x\right)=\sum_{j=0}^{5} a_{j}\left( \pm k_{i}\right)^{j}, \quad i=1,2,3$.

The solution of this system gives $a_{j}, j=0,1,2,3,4,5$. See the "Appendix".
Substituting from Eq. (32) into Eq. (33), we obtain the matrix exponential in the form

$\exp [A(s) \cdot x]=L(x, s)=\left[L_{i j}(x, s)\right], \quad i, j=1,2,3,4,5,6$,

where $L_{i j}(x, s), i, j=1,2,3,4,5,6$ are defined in the "Appendix".

Now, we will consider the first end of the nanobeams $x=0$ is clamped and loaded thermally, which gives (Youssef and Elsibai 2010):

$w(0, t)=\eta(0, t)=0$,

and

$\theta(0, t)=\theta_{0} f(t)$

where $\theta_{0}$ is constant.

Substituting from Eq. (37) into the mapping in (7), we obtain

$\vartheta_{1}(0, t)=\frac{1}{K_{\mathrm{o}}} \int_{0}^{\theta_{0} f(t)} K(\xi) \mathrm{d} \xi$.

After using Laplace transform, the above conditions take the forms

$\bar{w}(0, s)=\bar{\eta}(0, s)=0$,

and

$\bar{\vartheta}_{1}(0, s)=G(s)$.

where

$G(s)=\frac{1}{K_{\mathrm{o}}} \int_{0}^{\infty}\left[\int_{0}^{\theta_{0} f(t)} K(\xi) \mathrm{d} \xi\right] \mathrm{e}^{-s t} \mathrm{~d} t$.

Applying the conditions (39) and (40) into Eq. (29), we obtain

$\bar{V}(0, s)=\left[\begin{array}{c}0 \\ G(s) \\ 0 \\ \bar{w}^{\prime}(0, s) \\ \bar{\vartheta}_{1}^{\prime}(0, s) \\ \bar{\eta}^{\prime}(0, s)\end{array}\right]$.

To get $\bar{w}^{\prime}(0, s), \bar{\vartheta}_{1}^{\prime}(0, s)$ and $\bar{\eta}^{\prime}(0, s)$, we will consider the other end of the beam $x=\ell$ is clamped and remains at zero increment of temperature as follows:

$w(\ell, t)=\vartheta_{1}(\ell, t)=\eta(\ell, t)=0$.

After using Laplace transform, we have $\bar{w}(\ell, s)=\bar{\vartheta}_{1}(\ell, s)=\bar{\eta}(\ell, s)=0$.

Hence, we obtain 


$$
\begin{aligned}
& {\left[\begin{array}{c}
\bar{w}^{\prime}(0, s) \\
\bar{\theta}_{1}^{\prime}(0, s) \\
\bar{\eta}^{\prime}(0, s)
\end{array}\right]} \\
& =-G(s)\left(\left[\begin{array}{lll}
L_{14}(\ell, s) & L_{15}(\ell, s) & L_{16}(\ell, s) \\
L_{24}(\ell, s) & L_{25}(\ell, s) & L_{26}(\ell, s) \\
L_{34}(\ell, s) & L_{35}(\ell, s) & L_{36}(\ell, s)
\end{array}\right]^{-1}\left[\begin{array}{l}
L_{12}(\ell, s) \\
L_{22}(\ell, s) \\
L_{32}(\ell, s)
\end{array}\right]\right) .
\end{aligned}
$$

After some simplifications by using MAPLE software, we get the final solutions in the Laplace transform domain as follows:

$$
\begin{aligned}
& \bar{w}(x, s)=\frac{\Delta \sinh \left(k_{1}(\ell-x)\right)}{\left(k_{1}^{2}-k_{2}^{2}\right)\left(k_{1}^{2}-k_{3}^{2}\right) \sinh \left(k_{1} \ell\right)} \\
& +\frac{\Delta \sinh \left(k_{2}(\ell-x)\right)}{\left(k_{2}^{2}-k_{1}^{2}\right)\left(k_{2}^{2}-k_{3}^{2}\right) \sinh \left(k_{2} \ell\right)} \\
& +\frac{\Delta \sinh \left(k_{3}(\ell-x)\right)}{\left(k_{3}^{2}-k_{1}^{2}\right)\left(k_{3}^{2}-k_{2}^{2}\right) \sinh \left(k_{3} \ell\right)}, \\
& \bar{\vartheta}(z, x, s)=-\frac{\alpha_{2} k_{1}^{2} \Delta \sin (p z) \sinh \left(k_{1}(\ell-x)\right)}{\left(k_{1}^{2}-\alpha_{1}\right)\left(k_{1}^{2}-k_{2}^{2}\right)\left(k_{1}^{2}-k_{3}^{2}\right) \sinh \left(k_{1} \ell\right)} \\
& -\frac{\alpha_{2} k_{2}^{2} \Delta \sin (p z) \sinh \left(k_{2}(\ell-x)\right)}{\left(k_{2}^{2}-\alpha_{1}\right)\left(k_{2}^{2}-k_{1}^{2}\right)\left(k_{2}^{2}-k_{1}^{2}\right) \sinh \left(k_{2} \ell\right)} \\
& -\frac{\alpha_{3} k_{2}^{2} \Delta \sin (p z) \sinh \left(k_{3}(\ell-x)\right)}{\left(k_{3}^{2}-\alpha_{1}\right)\left(k_{3}^{2}-k_{1}^{2}\right)\left(k_{3}^{2}-k_{2}^{2}\right) \sinh \left(k_{3} \ell\right)}, \\
& \bar{u}(z, x, s)=-\frac{z \Delta k_{1} \cosh \left(k_{1}(\ell-x)\right)}{\left(k_{1}^{2}-k_{2}^{2}\right)\left(k_{1}^{2}-k_{3}^{2}\right) \sinh \left(k_{1} \ell\right)} \\
& -\frac{z \Delta k_{2} \cosh \left(k_{2}(\ell-x)\right)}{\left(k_{2}^{2}-k_{1}^{2}\right)\left(k_{2}^{2}-k_{1}^{2}\right) \sinh \left(k_{2} \ell\right)} \\
& -\frac{z \Delta k_{3} \cosh \left(k_{3}(\ell-x)\right)}{\left(k_{3}^{2}-k_{1}^{2}\right)\left(k_{3}^{2}-k_{2}^{2}\right) \sinh \left(k_{3} \ell\right)} \text {, } \\
& \bar{e}(z, x, s)=\frac{z \Delta k_{1}^{2} \sinh \left(k_{1}(\ell-x)\right)}{\left(k_{1}^{2}-k_{2}^{2}\right)\left(k_{1}^{2}-k_{3}^{2}\right) \sinh \left(k_{1} \ell\right)} \\
& +\frac{z \Delta k_{2}^{2} \sinh \left(k_{2}(\ell-x)\right)}{\left(k_{2}^{2}-k_{1}^{2}\right)\left(k_{2}^{2}-k_{1}^{2}\right) \sinh \left(k_{2} \ell\right)} \\
& +\frac{z \Delta k_{3}^{2} \sinh \left(k_{3}(\ell-x)\right)}{\left(k_{3}^{2}-k_{1}^{2}\right)\left(k_{3}^{2}-k_{2}^{2}\right) \sinh \left(k_{3} \ell\right)},
\end{aligned}
$$

where $\Delta=\frac{G(s)}{\alpha_{1} \alpha_{2}}\left(\alpha_{1}-k_{1}^{2}\right)\left(\alpha_{1}-k_{2}^{2}\right)\left(\alpha_{1}-k_{3}^{2}\right)$.

To determine the function $K(\theta)$, we will consider the thermal conductivity depends on the temperature with linear function in the form

$K(\theta)=K_{\mathrm{o}}\left(1+K_{1} \theta\right)$,

where for the gold, we have $K_{1} \geq 0$ (Zhang 2007).

By using the mapping in (7), we get

$\vartheta_{1}=\theta+\frac{K_{1}}{2} \theta^{2}$ and

$\vartheta_{1}(0, t)=\theta_{0} f(t)+\frac{K_{1}}{2}\left(\theta_{0} f(t)\right)^{2}$.

where $\theta_{0}$ is constant.

For thermal shock problem, we will consider

$f(t)=\mathrm{H}(t)=\left\{\begin{array}{cc}1 & t>0 \\ 0 & t<0\end{array}\right\}$,

where $\mathrm{H}(t)$ is called the Heaviside unite step function; then we get

$\vartheta_{1}(0, t)=\theta_{0} \mathrm{H}(t)+\frac{K_{1}}{2}\left(\theta_{0} H(t)\right)^{2}$,

Hence, we obtain

$G(s)=\left(\theta_{0}+\frac{K_{1}}{2} \theta_{0}^{2}\right) \frac{1}{s}$,

After obtaining $\vartheta$, the temperature increment $\theta$ can be obtained by solving Eq. (51) to get

$\theta=\frac{-1+\sqrt{1+2 K_{1} \vartheta}}{K_{1}} \quad$ where $\left(1+2 K_{1} \vartheta\right)>0$.

\section{The Strain-stress energy}

The stress on the $x$ axis, according to Hooke's law is

$\sigma_{x x}(x, z, t)=E\left(e-\alpha_{T} \theta\right)$.

By using the non-dimensional variables in (18), we obtain the stress in the form

$\sigma_{x x}(x, z, t)=e-\alpha_{T} T_{0} \theta$.

After using Laplace transform, the above equation takes the following form:

$\bar{\sigma}_{x x}(x, z, s)=\bar{e}-\alpha_{T} T_{0} \bar{\theta}$.

The strain energy which is generated on the beam is given by

$W(x, z, t)=\sum_{i, j=1}^{3} \frac{1}{2} \sigma_{i j} e_{i j}=\frac{1}{2} \sigma_{x x} e_{x x}=-\frac{1}{2} z \sigma_{x x} \eta$,

or, we can write it in the form

$W(x, z, t)=-\frac{1}{2} z\left[L^{-1}\left(\bar{\sigma}_{x x}\right)\right]\left[L^{-1}(\bar{\eta})\right]$,

where $L^{-1}[\cdot]$ is the inversion of Laplace transform.

\section{Numerical inversion of the Laplace transform}

In order to determine the solutions in the time domain, the Riemann-sum approximation method is used to obtain the 
numerical results. In this method, any function in Laplace domain can be inverted to the time domain as

$f(t)=\frac{e^{\eta t}}{t}\left[\frac{1}{2} \bar{f}(\eta)+\operatorname{Re} \sum_{n=1}^{N}(-1)^{n} \bar{f}\left(\eta+\frac{i n \pi}{t}\right)\right]$.

where $\mathrm{Re}$ is the real part and $i$ is imaginary number unit. For faster convergence, numerous numerical experiments have shown that the value of $\zeta$ satisfies the relation $\eta t \approx$ 4.7 Tzou (1996).

\section{Numerical results and discussion}

Now, we will consider a numerical example for which computational results are given. For this purpose, gold ( $\mathrm{Au}$ ) is taken as the thermoelastic material for which we take the following values of the different physical constants (Youssef and Elsibai 2010):

$$
\begin{aligned}
& K_{\mathrm{o}}=318 \mathrm{~W} /(\mathrm{mK}), \alpha_{T}=14.2(10)^{-6} \mathrm{~K}^{-1}, \\
& \rho=1,930 \mathrm{~kg} / \mathrm{m}^{3}, T_{0}=293 \mathrm{~K}, \\
& C_{v}=130 \mathrm{~J} /(\mathrm{kg} \mathrm{K}), E=180 \mathrm{GPa}, v=0.44 .
\end{aligned}
$$

The aspect ratios of the beam are fixed as $\ell / h=10$ and $b / h=1 / 2$, when $h$ is varied, and $\ell$ and $b$ change accordingly with $h$. For the nanoscale beam, we will take the range of the beam length $\ell(1-100) \times 10^{-9} \mathrm{~m}$, the original time $t$ will be considered in the picoseconds $(1-100) \times 10^{-12} \mathrm{sec}$ and the relaxation time $\tau_{\mathrm{o}}$ in the range $(1-100) \times 10^{-14} \mathrm{sec}$.

The figures were prepared using the non-dimensional variables which are defined in (18) for beam length $\ell=$ $1.0, \theta_{0}=1.0, z=h / 6, t=0.15$, and $K_{1}=0.0,0.2$ and 0.5.

The Figs. 2, 3, 4, 5, 6, 7 show that the variability of the thermal conductivity plays a vital role on the speed of the wave propagation of all the studied fields, and increasing of

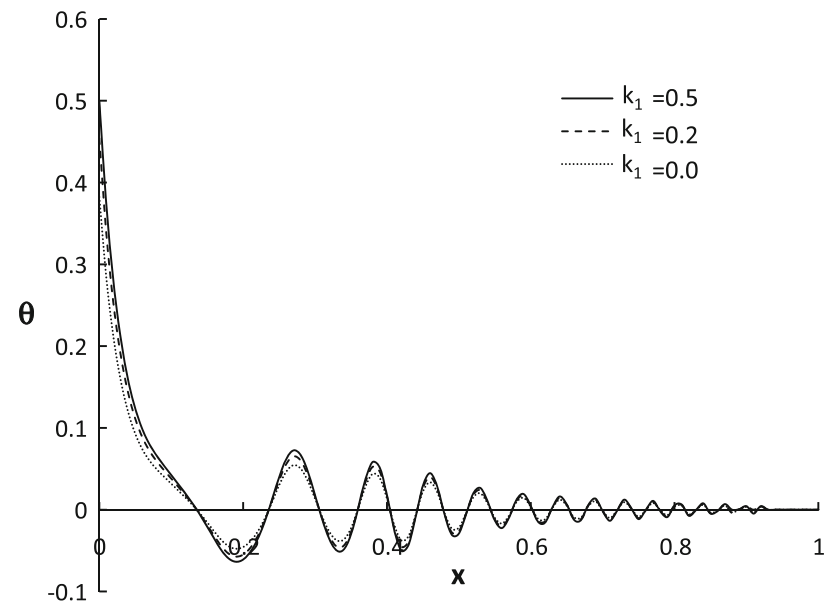

Fig. 2 The temperature distribution

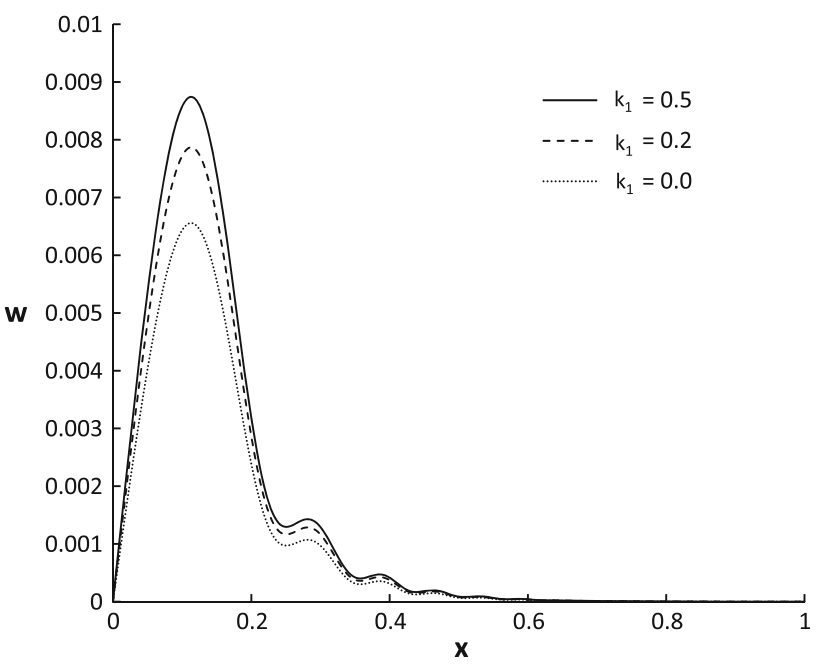

Fig. 3 The lateral vibration distribution

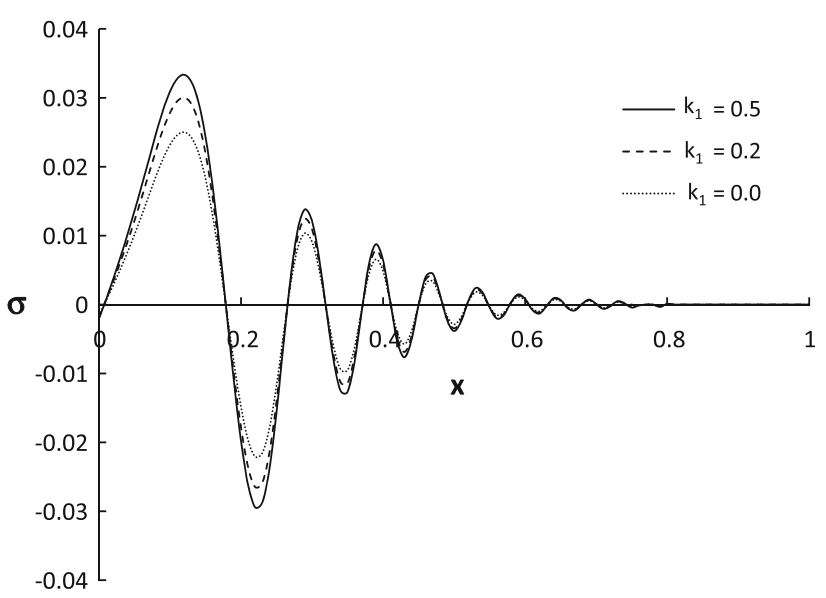

Fig. 4 The stress distribution

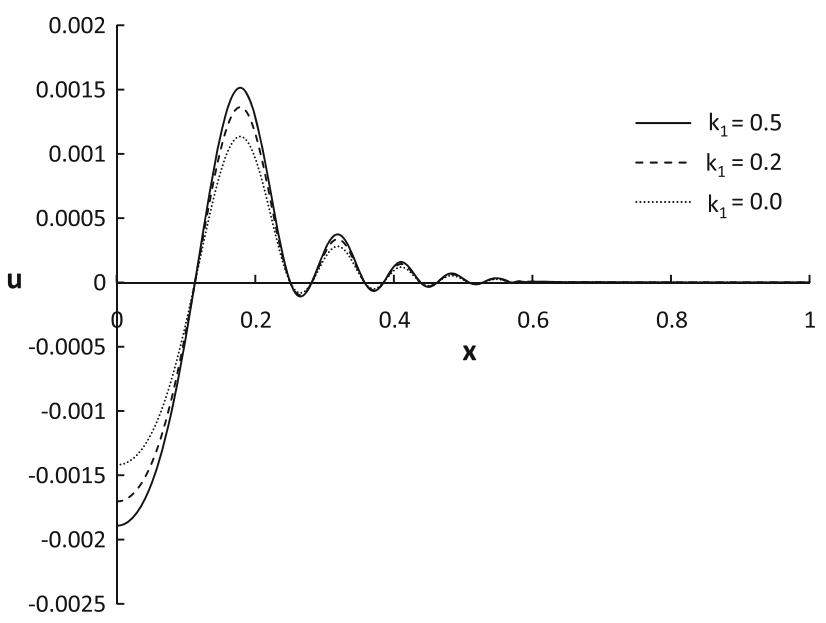

Fig. 5 The displacement distribution 


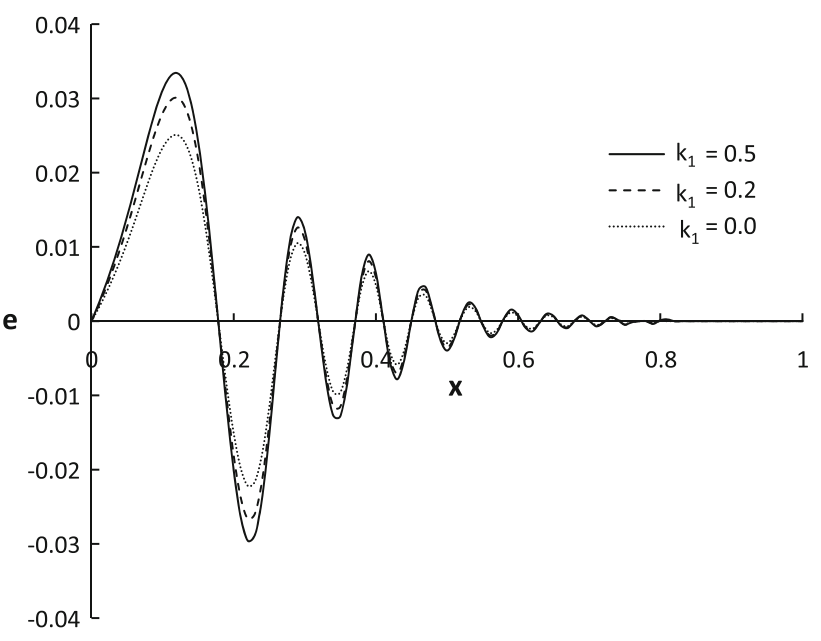

Fig. 6 The strain distribution

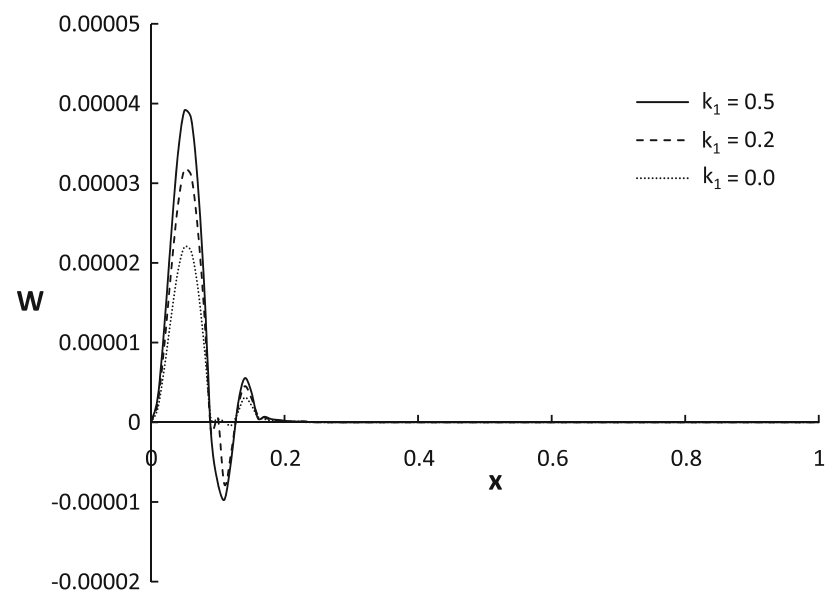

Fig. 7 The strain-stress energy distribution

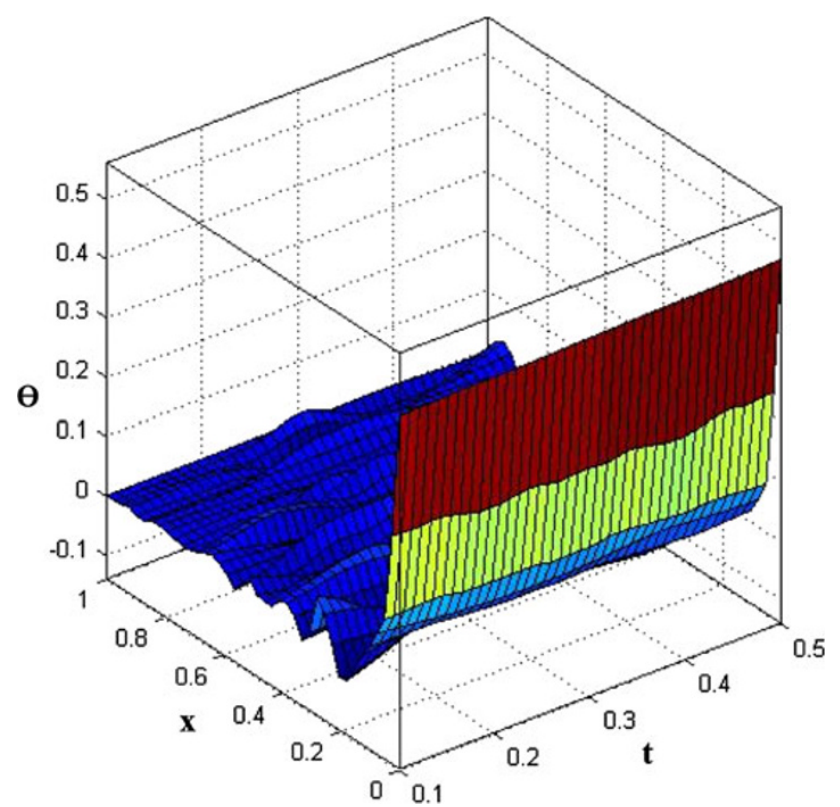

Fig. 8 The temperature distribution at $K_{1}=0.2$

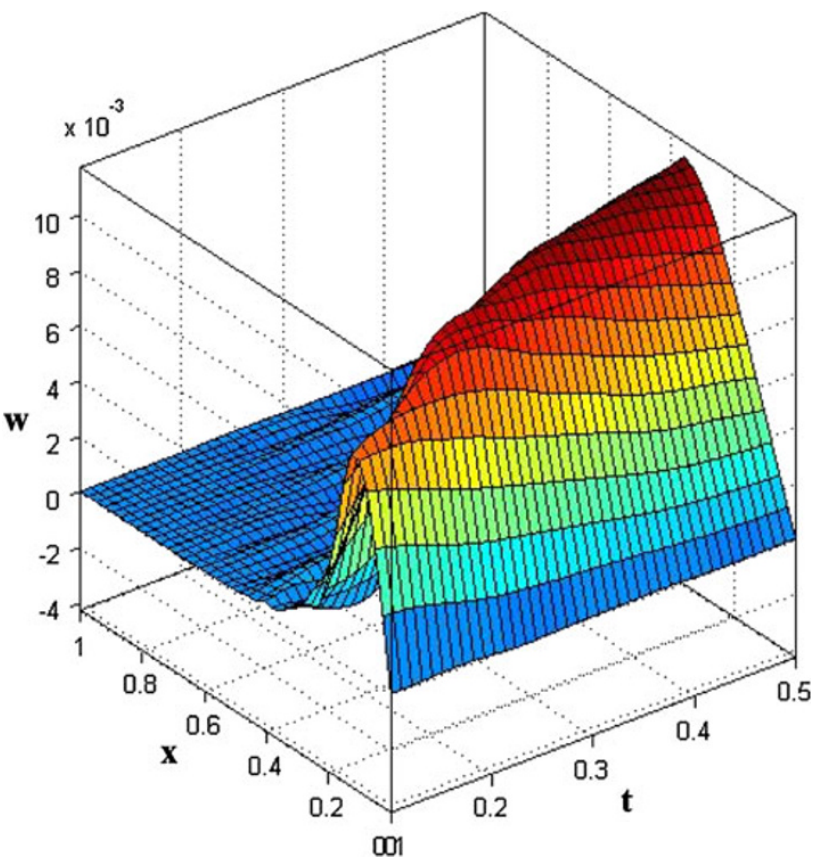

Fig. 9 The lateral vibration distribution at $K_{1}=0.2$

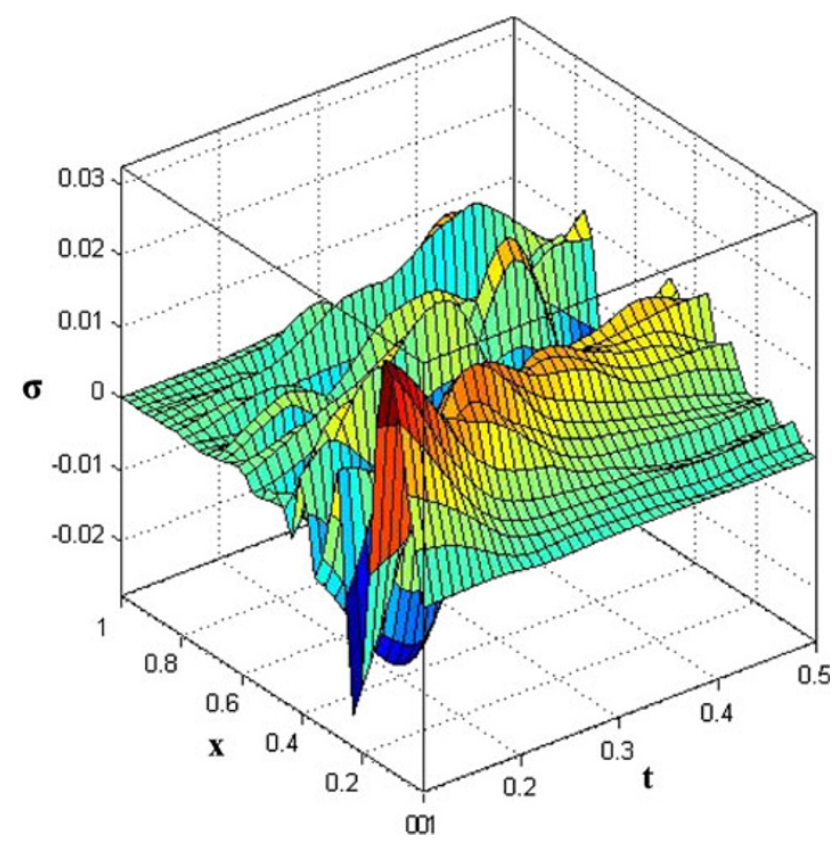

Fig. 10 The stress distribution at $K_{1}=0.2$

the parameter $K_{1}$ causes increasing on all the state functions distributions. The damping of the strain-stress energy decreases when the parameter $K_{1}$ increases.

Also, when $K_{1}=0.0$ all the results coincide with the results on (Youssef and Elsibai 2010).

Figures 8, 9, 10, 11, 12 and 13 represent the temperature, the lateral vibration, the stress, the displacement, the volumetric strain, and the stain-stress energy distribution when 


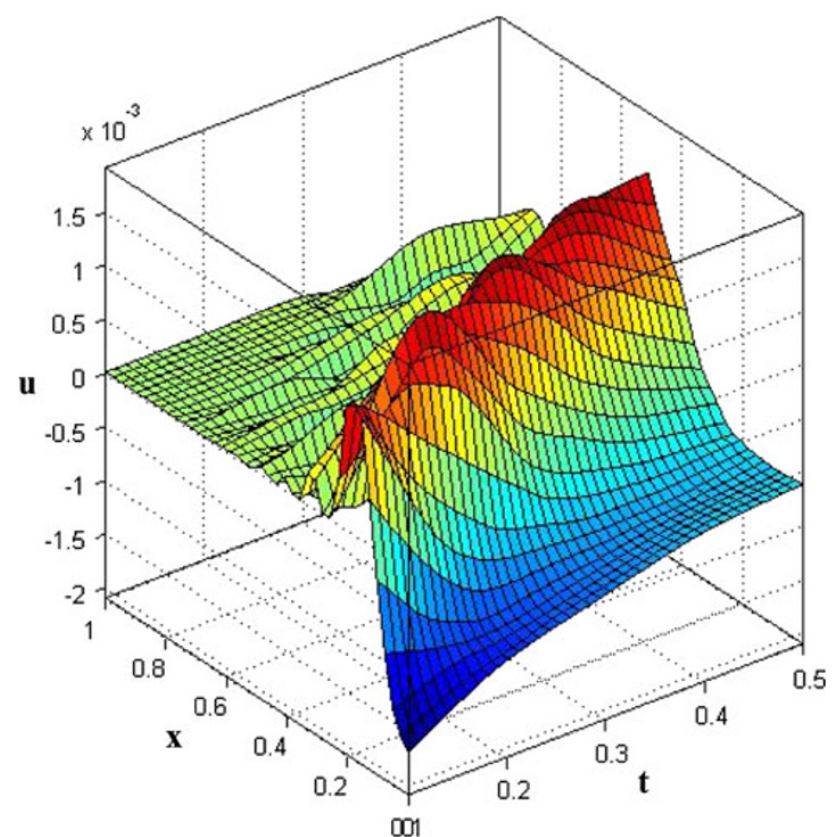

Fig. 11 The displacement distribution at $K_{1}=0.2$

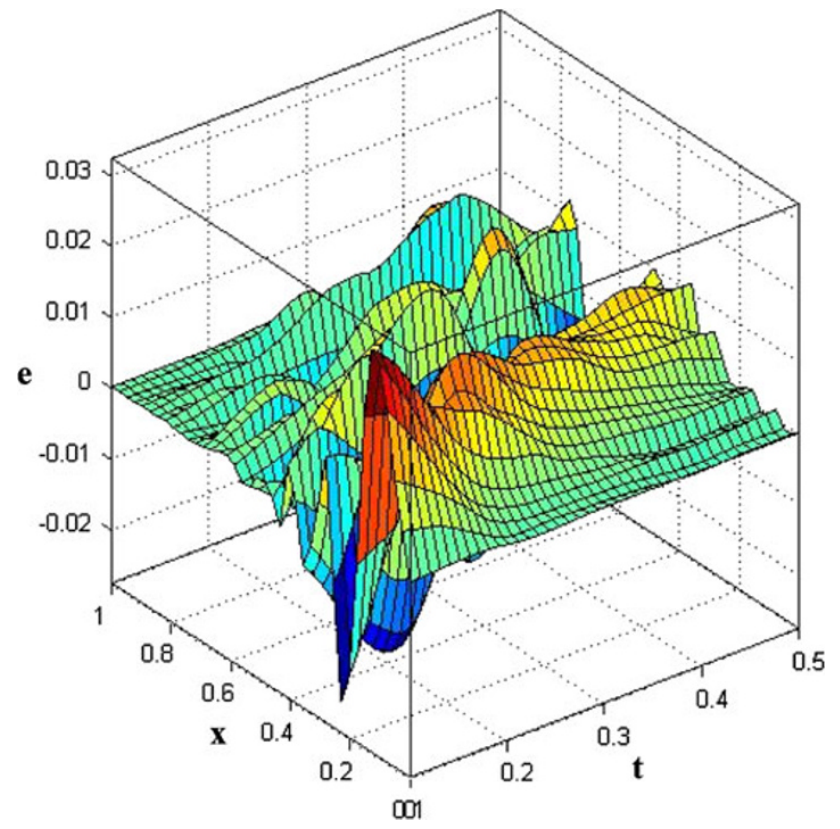

Fig. 12 The strain distribution at $K_{1}=0.2$

$K_{1}=0.2$ in three-dimension figures with wide range of the coordinate $x(0.0 \leq x \leq \ell)$ and the time $t(0.0<t \leq 0.5)$ to stand on the behavior of the wave propagation of all the studied fields with respect to the coordinate $x$ and the time $\mathrm{t}$ together. The peak points of the temperature, the lateral vibration, and the displacement increase when the time increases, whereas the strain, the stress, and the strain-stress energy decrease when the time increases.

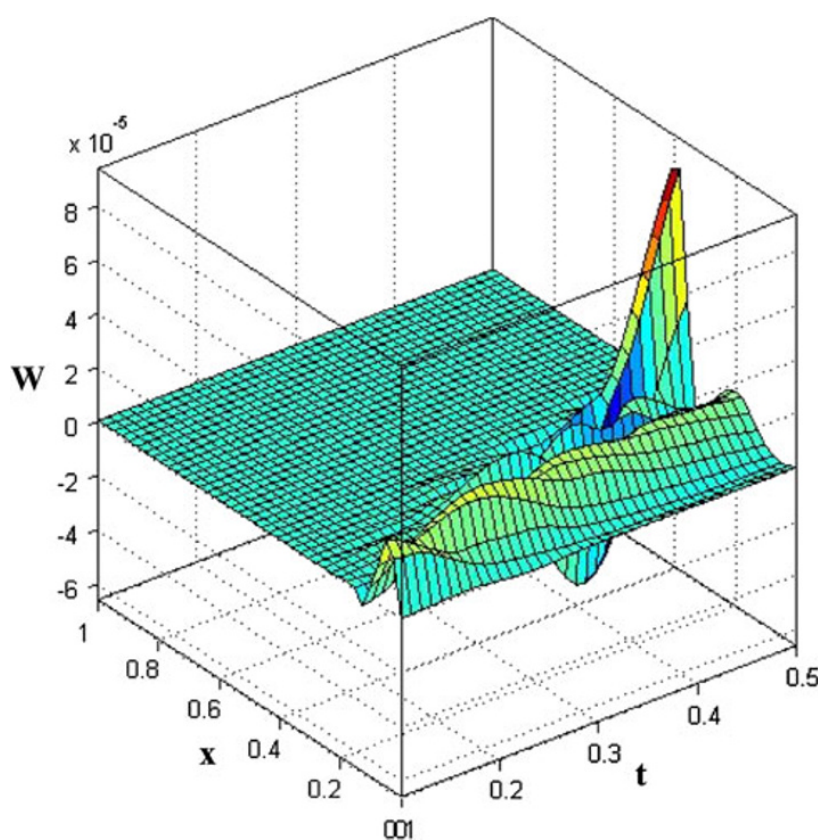

Fig. 13 The strain-stress energy distribution at $K_{1}=0.2$

\section{Conclusion}

This paper is dealing with the effect of the thermal conductivity on vibration of the deflection, the temperature, the displacement, the strain, the displacement, the stress, and the strain-stress energy. Euler-Bernoulli gold nanobeam with variable thermal conductivity induced by thermal shock has been considered. State-space approach and numerical technique, based on Laplace transformation, have been used to calculate the numerical results of all the studied fields. We get the following results:

1. Thermal conductivity has significant effects on the speed of the wave propagation of all the studied fields.

2. Thermal conductivity depends on the temperature with linear function in the form $K(\theta)=K_{\mathrm{o}}\left(1+K_{1} \theta\right)$ where for gold $K_{1} \geq 0$

3. The effect of the variability of the thermal conductivity decreases when the length of the beam increases.

4. The maximum value of the strain-stress energy increases when the changing in the thermal conductivity with respect to the temperature increases.

5. The changing in the thermal conductivity with respect to the temperature effects on the damping of the strain-stress energy of the beam.

Open Access This article is distributed under the terms of the Creative Commons Attribution License which permits any use, distribution, and reproduction in any medium, provided the original author(s) and the source are credited. 


\section{Appendix}

$$
\begin{aligned}
& F=\frac{1}{\left(k_{1}^{2}-k_{2}^{2}\right)\left(k_{2}^{2}-k_{3}^{2}\right)\left(k_{3}^{2}-k_{1}^{2}\right)}, \\
& \begin{array}{c}
c_{1}(x, s)=\left(k_{2}^{2}-k_{3}^{2}\right) \cosh \left(k_{1} x\right), c_{2}(x, s) \\
\quad=\left(k_{3}^{2}-k_{1}^{2}\right) \cosh \left(k_{2} x\right), \\
c_{3}(x, s)=\left(k_{1}^{2}-k_{2}^{2}\right) \cosh \left(k_{3} x\right), s_{1}(x, s) \\
=\frac{\left(k_{2}^{2}-k_{3}^{2}\right)}{k_{1}} \sinh \left(k_{1} x\right), \\
s_{2}(x, s)=\frac{\left(k_{3}^{2}-k_{1}^{2}\right)}{k_{2}} \sinh \left(k_{2} x\right), s_{3}(x, s) \\
=\frac{\left(k_{1}^{2}-k_{2}^{2}\right)}{k_{3}} \sinh \left(k_{3} x\right) .
\end{array}
\end{aligned}
$$$$
a_{0}(x, s)=-F\left(k_{2}^{2} k_{3}^{2} c_{1}+k_{1}^{2} k_{3}^{2} c_{2}+k_{1}^{2} k_{2}^{2} c_{3}\right),
$$$$
a_{1}(x, s)=-F\left(k_{2}^{2} k_{3}^{2} s_{1}+k_{1}^{2} k_{3}^{2} s_{2}+k_{1}^{2} k_{2}^{2} s_{3}\right),
$$$$
a_{2}(x, s)=F\left[\left(k_{2}^{2}+k_{3}^{2}\right) c_{1}+\left(k_{3}^{2}+k_{1}^{2}\right) c_{2}+\left(k_{1}^{2}+k_{2}^{2}\right) c_{3}\right] \text {, }
$$$$
a_{3}(x, s)=F\left[\left(k_{2}^{2}+k_{3}^{2}\right) s_{1}+\left(k_{3}^{2}+k_{1}^{2}\right) s_{2}+\left(k_{1}^{2}+k_{2}^{2}\right) s_{3}\right] \text {, }
$$$$
a_{4}(x, s)=-F\left(c_{1}+c_{2}+c_{3}\right), a_{5}(x, s)=-F\left(s_{1}+s_{2}+s_{3}\right) .
$$$$
L_{11}(x, s)=a_{0}-a_{4} \alpha_{3}, L_{12}(x, s)=-a_{4} \alpha_{4}, L_{13}(x, s)
$$$$
=a_{2}+a_{4} \alpha_{5} \text {, }
$$$$
L_{14}(x, s)=a_{1}-a_{5} \alpha_{3}, L_{15}(x, s)=-a_{5} \alpha_{4}, L_{16}(x, s)
$$$$
=a_{3}+a_{5} \alpha_{5} \text {, }
$$$$
L_{21}(x, s)=a_{4} \alpha_{2} \alpha_{3}, L_{22}(x, s)
$$$$
=a_{0}+a_{2} \alpha_{1}+a_{4}\left(\alpha_{1}^{2}+\alpha_{2} \alpha_{4}\right),
$$$$
L_{23}(x, s)=-a_{2} \alpha_{2}-a_{4} \alpha_{2}\left(\alpha_{1}+\alpha_{5}\right), L_{24}(x, s)=a_{5} \alpha_{2} \alpha_{3} \text {, }
$$$$
L_{25}(x, s)=a_{1}+a_{3} \alpha_{1}+a_{5}\left(\alpha_{1}^{2}+\alpha_{2} \alpha_{4}\right), L_{26}(x, s)
$$$$
=-a_{3} \alpha_{2}-a_{5} \alpha_{2}\left(\alpha_{1}+\alpha_{5}\right) \text {, }
$$$$
L_{31}(x, s)=-a_{2} \alpha_{3}-a_{4} \alpha_{3} \alpha_{5}, L_{32}(x, s)
$$$$
=-a_{2} \alpha_{4}-a_{4} \alpha_{4}\left(\alpha_{l}+\alpha_{5}\right),
$$

$$
L_{33}(x, s)=a_{0}+a_{2} \alpha_{5}+a_{4}\left(\alpha_{2} \alpha_{4}-\alpha_{3}+\alpha_{5}^{2}\right), L_{34}(x, s)
$$$$
=-a_{3} \alpha_{3}-a_{5} \alpha_{3} \alpha_{5} \text {, }
$$

$$
L_{35}(x, s)=-a_{3} \alpha_{4}-a_{5} \alpha_{4}\left(\alpha_{1}+\alpha_{5}\right), L_{36}(x, s)
$$$$
=a_{1}+a_{3} \alpha_{5}+a_{5}\left(\alpha_{2} \alpha_{4}-\alpha_{3}+\alpha_{5}^{2}\right),
$$

$$
\begin{aligned}
L_{41}(x, s) & =-a_{3} \alpha_{3}-a_{5} \alpha_{3} \alpha_{5}, L_{42}(x, s) \\
& =-a_{3} \alpha_{4}-a_{5} \alpha_{4}\left(\alpha_{1}+\alpha_{5}\right),
\end{aligned}
$$

$$
\begin{aligned}
L_{43}(x, s) & =a_{1}+a_{3} \alpha_{5}+a_{5}\left(\alpha_{2} \alpha_{4}-\alpha_{3}+\alpha_{5}^{2}\right), L_{44}(x, s) \\
& =a_{0}-a_{4} \alpha_{3}, L_{45}(x, s)=-a_{4} \alpha_{4},
\end{aligned}
$$

$$
\begin{aligned}
L_{46}(x, s) & =a_{2}+a_{4} \alpha_{5}, L_{51}(x, s) \\
& =a_{3} \alpha_{2} \alpha_{3}+a_{5} \alpha_{2} \alpha_{3}\left(\alpha_{1}+\alpha_{5}\right),
\end{aligned}
$$

$$
L_{52}(x, s)=a_{1} \alpha_{1}+a_{3}\left(\alpha_{1}^{2}+\alpha_{2} \alpha_{4}\right)+a_{5}\left(\alpha_{1}^{3}+2 \alpha_{1} \alpha_{2} \alpha_{4}\right.
$$$$
\left.+\alpha_{2} \alpha_{4} \alpha_{5}\right) \text {, }
$$

$$
\begin{aligned}
L_{53}(x, s)= & -a_{1} \alpha_{2}-a_{3} \alpha_{2}\left(\alpha_{1}+\alpha_{5}\right)-a_{5} \alpha_{2}\left(\alpha_{1}^{2}+\alpha_{1} \alpha_{5}\right. \\
& \left.+\alpha_{2} \alpha_{4}-\alpha_{3}+\alpha_{5}^{2}\right) \\
L_{54}(x, s)= & a_{4} \alpha_{2} \alpha_{3}, L_{55}(x, s)=a_{0}+a_{2} \alpha_{1}+a_{4}\left(\alpha_{1}^{2}+\alpha_{2} \alpha_{4}\right), \\
L_{56}(x, s)= & -a_{2} \alpha_{2}-a_{4} \alpha_{2}\left(\alpha_{1}+\alpha_{5}\right), L_{61}(x, s) \\
= & -a_{1} \alpha_{3}-a_{3} \alpha_{3} \alpha_{5}-a_{5} \alpha_{3}\left(\alpha_{2} \alpha_{4}-\alpha_{3}+\alpha_{5}^{2}\right) \\
L_{62}(x, s)= & -a_{1} \alpha_{4}-a_{3} \alpha_{4}\left(\alpha_{1}+\alpha_{5}\right)-a_{5} \alpha_{4}\left(\alpha_{1}^{2}+\alpha_{1} \alpha_{5}\right. \\
& \left.+\alpha_{2} \alpha_{4}-\alpha_{3}+\alpha_{5}^{2}\right) \\
L_{63}(x, s)= & a_{1} \alpha_{5}+a_{3}\left(\alpha_{2} \alpha_{4}-\alpha_{3}+\alpha_{5}^{2}\right)+a_{5}\left(\alpha_{1} \alpha_{2} \alpha_{4}\right. \\
& \left.+\alpha_{5}\left(2 \alpha_{2} \alpha_{4}-2 \alpha_{3}+\alpha_{5}^{2}\right)\right) \\
L_{64}(x, s)= & -a_{2} \alpha_{3}-a_{4} \alpha_{3} \alpha_{5} \\
L_{65}(x, s)= & -a_{2} \alpha_{4}-a_{4} \alpha_{4}\left(\alpha_{1}+\alpha_{5}\right) \\
L_{66}(x, s)= & a_{0}+a_{2} \alpha_{5}+a_{4}\left(\alpha_{2} \alpha_{4}-\alpha_{3}+\alpha_{5}^{2}\right)
\end{aligned}
$$

\section{References}

Al-Huniti NS, Al-Nimr MA, Naij M (2001) Dynamic response of a rod due to a moving heat source under the hyperbolic heat conduction model. J Sound Vib 242:629-640

Bahar LY, Hetnarski RB (1977) State space approach to thermoelasticity. In: Proceedings of 6th Canadian Congress of Applied Mechanics, University of British Columbia, Vancouver, British Columbia, Canada, pp 17-18

Bahar LY, Hetnarski RB (1977) Transfer matrix approach to thermoelasticity. In: Proceedings of 15 th Midwest mechanical conference, University of Illinois at Chicago Circle, pp 161-163

Bahar LY, Hetnarski RB (1978) State space approach to thermoelasticity. J Therm Stresses 1:135-145

Biot M (1956) Thermoelasticity and irreversible thermo-dynamics. J Appl Phys 27:240-253

Boley BA (1972) Approximate analyses of thermally induced vibrations of beams and plates. J Appl Mech 39:212-216

Diao JK, Gall K, Dunn ML (2004) Atomistic simulation of the structure and elastic properties of gold nanowires. J Mech Phys Solids 52:1935-1962

Duwel A, Gorman J, Weinstein M, Borenstein J, Ward P (2003) Experimental study of thermoelastic damping in MEMS gyros. Sens Actuators, A 103:70-75

Ezzat MA (2008) State space approach to solids and fluids (review). Can J Phys 86:1241-1250

Ezzat MA, Youssef HM (2009) Stat-space approach of conducting magneto-thermoelastic medium with variable thermal conductivity subjected to ramp-type heating. J Therm Stresses 32:414427

Fang DN, Sun YX, Soh AK (2006) Analysis of frequency spectrum of laser-induced vibration of microbeam resonators. Chin Phys Lett 23(6):1554-1557

Kidawa-Kukla J (2003) Application of the Green functions to the problem of the thermally induced vibration of a beam. J Sound Vib 262:865-876

Lord H, Shulman Y (1967) A generalized dynamical theory of thermoelasticity. Mech Phys Solid 15:299-309

Manolis GD, Beskos DE (1980) Thermally induced vibrations of beam structures. Comput Methods Appl Mech Eng 21:337-355

Rao JS (1992) Advanced theory of vibration (Nonlinear Vibration and One Dimensional Structures). Wiley, New York 
Soh AK, Sun Y, Fang D (2008) Vibration of microscale beam induced by laser pulse. J Sound Vib 311:243-253

Sun YX, Fang DN, Soh AK (2006) Thermoelastic damping in microbeam resonators. Int J Solids Struct 43:3213-3229

Sun Y, Fang D, Saka M, Soh AK (2008) Laser-induced vibrations of micro-beams under different boundary conditions. Int J Solids Struct 45:1993-2013

Tzou D (1996) Macro-to -micro heat transfer. Taylor \& Francis, Washington DC

Wang YZ, Li FM, Kishimoto K (2010) Scale effects on the longitudinal wave propagation in nanoplates. Physica E 42: $1356-1360$

Youssef HM, El-Bary AA (2006a) Thermal shock problem of a generalized thermoelastic layered composite material with variable thermal conductivity. J Math Prob Eng 87940:1-14
Youssef HM, El-Bary AA (2006b) Mathematical model for thermal shock problem of a generalized thermoelastic layered composite material with variable thermal conductivity. J Comput Methods Sci Technol 12(2):165-171

Youssef HM, El-Bary AA (2006c) Generalized thermoelasticity for an infinite material with variable thermal conductivity. J Adv Math Sci Appl 16(1):339-353

Youssef HM, Elsibai KA (2010) Vibration of gold nano-beam induced by different types of thermal loading- a state-space approach. J Nanoscale Microscale Thermophys Eng 15(1):48-69

Zhang ZM (2007) Nano/Microscale heat transfer, McGraw-Hill, USA. doi:10.1036/007143674X 